\title{
IMA Commission on New Minerals, Nomenclature and Classification (CNMNC)
}

\section{Newsletter 54}

\author{
Ritsuro Miyawaki (Chairman, CNMNC) ${ }^{1}$, Frédéric Hatert (Vice-Chairman, CNMNC) ${ }^{2}$, Marco Pasero (Vice-Chairman, \\ CNMNC) ${ }^{3 *}$ and Stuart J. Mills (Secretary, CNMNC) ${ }^{4}$ \\ ${ }^{1}$ Department of Geology and Paleontology, National Museum of Nature and Science, 4-1-1 Amakubo, Tsukuba 305-0005, Japan - miyawaki@kahaku.go.jp; \\ ${ }^{2}$ Laboratoire de Minéralogie, Université de Liège, B-4000 Liège, Belgium - fhatert@uliege.be; ${ }^{3}$ Dipartimento di Scienze della Terra, Università di Pisa, Via Santa Maria \\ 53, I-56126 Pisa, Italy - marco.pasero@unipi.it; and ${ }^{4}$ Geosciences, Museums Victoria, PO Box 666, Melbourne, Victoria 3001, Australia - smills@museum.vic.gov.au
}

The information given here is provided by the IMA Commission on New Minerals, Nomenclature and Classification for comparative purposes and as a service to mineralogists working on new species.

Each mineral is described in the following format:

Mineral name, if the authors agree on its release prior to the full description appearing in press

Chemical formula

Type locality

Full authorship of proposal

E-mail address of corresponding author

Relationship to other minerals

Crystal system, Space group; Structure determined, yes or no

Unit-cell parameters

Strongest lines in the powder X-ray diffraction pattern

Type specimen repository and specimen number

Citation details for the mineral prior to publication of full description

Citation details concern the fact that this information will be published in the Mineralogical Magazine on a routine basis, as well as being added month by month to the Commission's website.

It is still a requirement for the authors to publish a full description of the new mineral.

\section{NO OTHER INFORMATION WILL BE RELEASED BY THE COMMISSION}

\section{NEW MINERAL PROPOSALS APPROVED IN FEBRUARY 2020}

IMA No. 2018-121a

Pošepnýite

$\left(\mathrm{Cu}_{3}^{+} \square_{3}\right)_{\Sigma 6}\left(\mathrm{Hg}_{4}^{2+} \mathrm{Cu}_{2}^{+}\right)_{\Sigma 6} \mathrm{Sb}_{4}\left(\mathrm{Se}_{12.5} \square_{0.5}\right)_{\Sigma 13}$

At the mine dump of shaft No. 16 - Háje, near Př́bram, Central

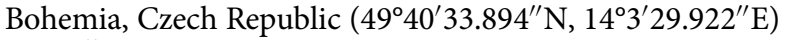

Pavel Škácha*, Jiři Sejkora, Jakub Plášil and Emil Makovicky

${ }^{\star}$ E-mail: skacha-p@muzeum-pribram.cz

Tetrahedrite group

Cubic: $I \overline{4} 3 m$; structure determined

$a=10.964(1) \AA$

4.476(11), 3.165(100), 2.930(24), 2.002(16), $1.938(65), 1.778$

(13), 1.653(31), 1.119(12)

Type material is deposited in the mineralogical collections of the National Museum, Department of Mineralogy and Petrology, Cirkusová 1740, Praha 9, Czech Republic, catalogue number P1P 15/2015, and the Mining Museum, Hynka Kličky

\footnotetext{
*Author for correspondence: Marco Pasero, Email: marco.pasero@unipi.it Cite this article: Miyawaki R., Hatert F., Pasero M. and Mills S.J. (2020) Newsletter 54. Mineralogical Magazine 84, 359-365. https://doi.org/10.1180/mgm.2020.21
}

Place 293, Příbram VI, Březové Hory, Czech Republic, catalogue number $1 / 2016$

How to cite: Škácha, P., Sejkora, J., Plášil, J. and Makovicky, E. (2020) Pošepnýite, IMA 2018-121a. CNMNC Newsletter No. 54; Mineralogical Magazine, 84, https://doi.org/10.1180/ mgm.2020.21

IMA No. 2018-145

Hydroxyplumbopyrochlore

$\left(\mathrm{Pb}_{1.5} \square_{0.5}\right) \mathrm{Nb}_{2} \mathrm{O}_{6}(\mathrm{OH})$

Jabal Sayid peralkaline granitic complex, Saudi Arabia $\left(23^{\circ} 49^{\prime} 28.72^{\prime \prime} \mathrm{N}, 40^{\circ} 56^{\prime} 30.93^{\prime \prime} \mathrm{E}\right)$

Ting $\mathrm{Li}^{\star}$, Ziying Li, Guang Fan, Honghai Fan, Jun Zhong, Naser S. Jahdali, Mingkuan Qin, Abdullah M. Jehani, Fenggang Wang and Mubarak M. Nahdi

*E-mail: liting_c@126.com

Pyrochlore supergroup

Cubic: $F d \overline{3} m$; structure determined

$a=10.558(2) \AA$

6.051(8), 3.043(100), 2.636(42), 1.862(36), 1.591(43), 1.521(8), $1.320(6), 1.183(12)$ 
Type material is deposited in the mineralogical collections of the Geological Museum of China, No. 16 Yangrou Hutong, Xisi, Beijing 100031, People's Republic of China, catalogue number M13239

How to cite: Li, T., Li, Z., Fan, G., Fan, H., Zhong, J., Jahdali, N.S., Qin, M., Jehani, A.M., Wang, F. and Nahdi, M.M. (2020) Hydroxyplumbopyrochlore, IMA 2018-145. CNMNC Newsletter No. 54; Mineralogical Magazine, 84, https://doi. org/10.1180/mgm.2020.21

\section{IMA No. 2019-092}

Stangersite

$\mathrm{SnGeS}_{3}$

In the central part of a burning coal mine dump of the abandoned Kateřina mine, Radvanice village, ca. $12 \mathrm{~km} \mathrm{E}$ of Trutnov, northern Bohemia, Czech Republic $\left(50^{\circ} 33^{\prime} 39.0^{\prime \prime} \mathrm{N}\right.$, $\left.16^{\circ} 03^{\prime} 56.2^{\prime \prime} \mathrm{E}\right)$

Jiří Sejkora*, Emil Makovicky, Tonci Balić-Žunić and Peter Berlepsch

^E-mail: jiri_sejkora@nm.cz

Known synthetic analogue

Monoclinic: $P 2_{1} / c$; structure determined

$a=7.270(1), b=10.197(2), c=6.846(1) \AA, \beta=105.34(3)^{\circ}$

7.006(100), 4.135(49), 3.077(47), 2.810(29), 2.776(38), 2.699 (69), 2.121(31), 1.724(35)

Type material is deposited in the mineralogical collections of the Department of Mineralogy and Petrology, National Museum in Prague, Cirkusová 1740, 19300 Praha 9, Czech Republic, catalogue number P1P 8/2000

How to cite: Sejkora, J., Makovicky, E., Balić-Žunić, T. and Berlepsch, P. (2020) Stangersite, IMA 2019-092. CNMNC Newsletter No. 54; Mineralogical Magazine, 84, https://doi. org/10.1180/mgm.2020.21

\section{IMA No. 2019-101}

Meifuite

$\mathrm{KFe}_{6}\left(\mathrm{Si}_{7} \mathrm{Al}\right) \mathrm{O}_{19}(\mathrm{OH})_{4} \mathrm{Cl}_{2}$

$\mathrm{Fe}-\mathrm{Cu}$ ore deposit of Yinachang, Yangtze Block, China

Shiyun Jin, Xiaochun $\mathrm{Li}$ and Huifang $\mathrm{Xu}^{*}$

^E-mail: hfxu@geology.wisc.edu

Chemically and structurally related to annite

Triclinic: $P \overline{1}$; structure determined

$a=22.777(1), b=9.5553(5), c=14.3282(8) \AA, \alpha=99.258(4)$,

$\beta=136.750(3), \gamma=89.899(4)^{\circ}$

9.56(100), 4.78(25), 3.19(19), 2.79(20), 2.69(15), 2.55(37), 2.44

(13), 2.23(15)

Type material is deposited in the mineralogical collections of the Geology Museum, Department of Geoscience, University of Wisconsin, 1215 West Dayton Street, Madison, WI 53706, USA, catalogue numbers UWGM 6859 (holotype), UWGM 6860 and UWGM 6861 (cotypes)

How to cite: Jin, S., Li, X. and Xu, H. (2020) Meifuite, IMA 2019-101. CNMNC Newsletter No. 54; Mineralogical Magazine, 84, https://doi.org/10.1180/mgm.2020.21

\section{IMA No. 2019-102}

Dutkevichite-(Ce)

$\mathrm{NaZnBa}_{2} \mathrm{Ce}_{2} \mathrm{Ti}_{2} \mathrm{Si}_{8} \mathrm{O}_{26} \mathrm{~F} \cdot \mathrm{H}_{2} \mathrm{O}$

In the moraine of Darai-Pioz glacier, Alai mountain range, Tien-Shan, Rashtskii district, Tajikistan $\left(39^{\circ} 30^{\prime} \mathrm{N}, 70^{\circ} 40^{\prime} \mathrm{E}\right)$

Atali A. Agakhanov*, Leonid A. Pautov, Natalia V. Zubkova, Andrey A. Zolotarev, Anatoly V. Kasatkin, Vladimir
Y. Karpenko, Igor V. Pekov, Vitaliya A. Agakhanova, Vyacheslav A. Muftakhov, Radek Škoda and Sergey N. Britvin ^E-mail: atali99@mail.ru

The $\mathrm{Zn}$ analogue of byelorussite-(Ce)

Orthorhombic: Ama2; structure determined

$a=22.238(5), b=10.497(3), c=9.649(2) \AA$

4.420(73), 4.281(35), 3.287(53), 3.203(39), 2.992(100), 2.939

(56), 2.779(47), 2.606(40)

Type material is deposited in the collections of the Fersman Mineralogical Museum, Russian Academy of Sciences, Leninskiy Prospekt 18-2, Moscow 119071, Russia, registration number 5281/1

How to cite: Agakhanov, A.A., Pautov, L.A., Zubkova, N.V., Zolotarev, A.A., Kasatkin, A.V., Karpenko, V.Y., Pekov, I.V., Agakhanova, V.A., Muftakhov, V.A., Škoda, R. and Britvin, S.N. (2020) Dutkevichite-(Ce), IMA 2019-102. CNMNC Newsletter No. 54; Mineralogical Magazine, 84, https://doi. org/10.1180/mgm.2020.21

\section{IMA No. 2019-105}

Niasite

$\mathrm{Ni}_{4.5}^{2+}\left(\mathrm{AsO}_{4}\right)_{3}$

Johanngeorgenstadt, Erzgebirgskreis, Saxony, Germany

Anthony R. Kampf ${ }^{\star}$, Barbara P. Nash, Jakub Plášil, Jason B. Smith and Mark N. Feinglos

*E-mail: akampf@nhm.org

Isostructural with jeffbenite

Tetragonal: $I \overline{4} 2 d$; structure determined

$a=6.8046(8), c=18.619(1) \AA$

$3.274(18), 3.008(25), 2.752(100), 2.330(10), 1.706(21), 1.678$

(28), 1.446(13), 1.412(10)

Cotype material is deposited in the mineralogical collections of the Natural History Museum of Los Angeles County, 900 Exposition Boulevard, Los Angeles, CA 90007, USA, catalogue number 74203, and the American Museum of Natural History, Central Park West @ 79th Street, New York City, NY 10024, USA, catalogue number 17956

How to cite: Kampf, A.R., Nash, B.P., Plášil, J., Smith, J.B. and Feinglos, M.N. (2020) Niasite, IMA 2019-105. CNMNC Newsletter No. 54; Mineralogical Magazine, 84, https://doi. org/10.1180/mgm.2020.21

\section{IMA No. 2019-106}

Dobrovolskyite

$\mathrm{Na}_{4} \mathrm{Ca}\left(\mathrm{SO}_{4}\right)_{3}$

Second scoria cone of the Northern Breakthrough of the Great Tolbachik fissure eruption, Tolbachik volcano, Kamchatka, Far-Eastern Region, Russia $\left(55^{\circ} 41^{\prime} \mathrm{N}, 160^{\circ} 14^{\prime} \mathrm{E}, 1200 \mathrm{~m}\right.$ asl)

Andrey P. Shablinskii*, Stanislav K. Filatov, Lidiya P. Vergasova, Svetlana V. Moskaleva, Eugeniya Y. Avdontseva and Rimma S. Bubnova

*E-mail: shablinskii.andrey@mail.ru

Chemically and structurally related to bubnovaite

Trigonal: $R 3$; structure determined

$a=15.7223(2), c=22.0160(5) \AA$

11.58(40), 5.79(22), 3.86(88), 3.67(32), 3.11(24), 3.09(26), 2.855

(50), 2.682(100)

Type material is deposited in the collections of the Saint-Petersburg State University mineralogical museum, University Emb. 7/9, St. Petersburg 199034, Russia, catalogue number $1 / 19829$ 
How to cite: Shablinskii, A.P., Filatov, S.K., Vergasova, L.P., Moskaleva, S.V., Avdontseva, E.Y. and Bubnova, R.S. (2020) Dobrovolskyite, IMA 2019-106. CNMNC Newsletter No. 54; Mineralogical Magazine, 84, https://doi.org/10.1180/mgm. 2020.21

\section{IMA No. 2019-108}

Keplerite

$\mathrm{Ca}_{9}\left(\mathrm{Ca}_{0.5} \square_{0.5}\right) \mathrm{Mg}\left(\mathrm{PO}_{4}\right)_{7}$

Marjalahti meteorite, fell June 01, 1902 at the northern coast of Lake Ladoga, Karelia, Russia ( $61^{\circ} 30^{\prime} \mathrm{N}, 30^{\circ} 30^{\prime} \mathrm{E}$ - holotype); Hatrurim Formation, near Arad, Negev Desert, Israel $\left(31^{\circ} 13^{\prime} 58^{\prime \prime} \mathrm{N}, 35^{\circ}\right.$ $16^{\prime} 2^{\prime \prime} \mathrm{E}$ - cotype)

Sergey N. Britvin*, Irina O. Galuskina, Natalia S. Vlasenko, Oleg S. Vereshchagin, Vladimir N. Bocharov, Maria G. Krzhizhanovskaya, Vladimir V. Shilovskikh, Evgeny V. Galuskin, Yevgeny Vapnik and Edita V. Obolonskaya

*E-mail: sbritvin@gmail.com

The $\mathrm{Mg}$ analogue of matyhite

Trigonal: $R 3 c$; structure determined

$a=10.3330(4), c=37.067(2) \AA$

5.166(25), 3.424(24), 3.177(46), 2.854(100), 2.583(64), 1.915 (22), 1.877(19), 1.712(28)

Type material is deposited in the collections of the Mining Museum, Saint Petersburg Mining University, St. Petersburg, Russia, catalogue number MM74/2-1

How to cite: Britvin, S.N., Galuskina, I.O., Vlasenko, N.S., Vereshchagin, O.S., Bocharov, V.N., Krzhizhanovskaya, M.G., Shilovskikh, V.V., Galuskin, E.V., Vapnik, Y. and Obolonskaya, E.V. (2020) Keplerite, IMA 2019-108. CNMNC Newsletter No. 54; Mineralogical Magazine, 84, https://doi.org/10.1180/mgm. 2020.21

\section{IMA No. 2019-111}

Tilkerodeite

$\mathrm{Pd}_{2} \mathrm{HgSe}_{3}$

Eskaborn adit (60-m level, $5 \mathrm{~m} \mathrm{~N}$ of the blind shaft IV), Tilkerode, Harz Mountains, Germany $\left(51^{\circ} 38^{\prime} 3^{\prime \prime} \mathrm{N}, 11^{\circ} 19^{\prime} 4^{\prime \prime} \mathrm{E}\right)$

Chi $\mathrm{Ma}^{\star}$ and Hans-Jürgen Förster

${ }^{\star}$ E-mail: chi@gps.caltech.edu

The $\mathrm{Pd}$ analogue of jacutingaite

Trigonal: $P \overline{3} m 1$

$a=7.325, c=5.288 \AA$

5.288(9), 2.720(100), 2.031(34), 1.831(37), 1.519(19), 1.360(10), $1.270(10), 1.169(15)$

Type material is deposited in the collections of the Mineralogical Institute, Technische Universität Bergakademie Freiberg, Freiberg, Germany, inventory number MiSa84670

How to cite: Ma, C. and Förster, H.-J. (2020) Tilkerodeite, IMA 2019-111. CNMNC Newsletter No. 54; Mineralogical Magazine, 84, https://doi.org/10.1180/mgm.2020.21

\section{IMA No. 2018-073b}

Fleetite

$\mathrm{Cu}_{2} \mathrm{RhIrSb}_{2}$

Miass Placer Zone, close to the city of Miass, Chelyabinsk oblast, southern Urals, Russia

Andrei Y. Barkov*, Luca Bindi, Nobumichi Tamura, Robert F. Martin, Chi Ma, Björn Winkler, Gennadiy I. Shvedov and Wolfgang Morgenroth

${ }^{\star}$ E-mail: ore-minerals@mail.ru

New structure type
Cubic: $F d \overline{3} m$; structure determined

$a=11.6682(8) \AA$

6.7(75), 4.13(100), 3.52(30), 2.679(20), 2.380(50), 2.064(40), 1.969(15), 1.556(20)

Type material is deposited in the mineralogical collections of the State Museum of Geology of Central Siberia, 13 Partizana Zheleznyaka Street, 660021 Krasnoyarsk, Russia, catalogue number $1 / 41 / 11002$

How to cite: Barkov, A.Y., Bindi, L., Tamura, N., Martin, R.F., Ma, C., Winkler, B., Shvedov, G.I. and Morgenroth, W. (2020) Fleetite, IMA 2018-073b. CNMNC Newsletter No. 54; Mineralogical Magazine, 84, https://doi.org/10.1180/mgm. 2020.21

IMA No. 2018-132a

Chukochenite

$\mathrm{LiAl}_{5} \mathrm{O}_{8}$

Xianghualing skarn, Linwu County, Hunan Province, China $\left(25^{\circ} 26^{\prime} \mathrm{N}, 112^{\circ} 34^{\prime} \mathrm{E}\right)$

Can Rao*, Xiangping Gu, Rucheng Wang, Qunke Xia, Yuanfeng Cai, Chuanwan Dong, Frédéric Hatert and Yantao Hao

${ }^{\star}$ E-mail: canrao@zju.edu.cn

New structure type

Orthorhombic: Imma; structure determined

$a=5.642(1), b=16.827(2), c=8.014(1) \AA$

2.404(53), 1.538(77), 1.412(100), 1.256(52), $1.068(36), 1.040$

(61), 0.999(59), 0.942(35)

Type material is deposited in the mineralogical collections of the Geological Museum of China, No. 15 Yangrou Hutong, Xisi, Beijing 100031, People's Republic of China, catalogue number M13818

How to cite: Rao, C., Gu, X., Wang, R., Xia, Q., Cai, Y., Dong, C., Hatert, F. and Hao, Y. (2020) Chukochenite, IMA 2018-132a. CNMNC Newsletter No. 54; Mineralogical Magazine, 84, https://doi.org/10.1180/mgm.2020.21

\section{IMA No. 2018-026b}

Poirierite

$\mathrm{Mg}_{2} \mathrm{SiO}_{4}$

Suizhou meteorite, fell at Xihe, Zengdu District, Suizhou, Hubei, China $\left(31^{\circ} 37^{\prime} \mathrm{N}, 113^{\circ} 28^{\prime} \mathrm{E}\right.$ - holotype); Tenham meteorite, fell at Tenham Station, Windorah, Barcoo Shire, Queensland, Australia $\left(25^{\circ} 43^{\prime} 59^{\prime \prime} \mathrm{S}, 142^{\circ} 57^{\prime} 0^{\prime \prime} \mathrm{E}\right.$ - cotype); Miami meteorite, Texas, USA $\left(35^{\circ} 40^{\prime} \mathrm{N}, 100^{\circ} 36^{\prime} \mathrm{W}\right)$

Naotaka Tomioka*, Takuo Okuchi, Toshiaki Iitaka, Masaaki Miyahara, Luca Bindi and Xiande Xie

${ }^{\star}$ E-mail: tomioka@jamstec.go.jp

A polymorph of forsterite, wadsleyite and ringwoodite

Orthorhombic: Pmma; structure determined

$a=5.801(11), b=2.905(9), c=8.411(16) \AA$

3.405(54), 2.804(71), 2.742(62), 2.482(95), 2.053(82), 1.469 (100), 1.453(32), 1.450(32)

Type material is deposited in the mineralogical collections of the Museo di Storia Naturale, Università di Firenze, Via La Pira 4, I-50121, Firenze, Italy, catalogue number 3238/I (holotype - Suizhou), and the Department of Geology and Paleontology, National Museum of Nature and Science, Tokyo, Japan, sample number NSM-MF15125 (cotype Tenham)

How to cite: Tomioka, N., Okuchi, T., Iitaka, T., Miyahara, M., Bindi, L. and Xie, X. (2020) Poirierite, IMA 2018-026b. 
CNMNC Newsletter No. 54; Mineralogical Magazine, 84, https://doi.org/10.1180/mgm.2020.21

\section{NEW MINERAL PROPOSALS APPROVED IN MARCH 2020}

IMA No. 2019-084

Zaykovite

$\mathrm{Rh}_{3} \mathrm{Se}_{4}$

Kazan gold-bearing placer, Gogino placer zone, $100-150 \mathrm{~km}$ SSE from the city of Magnitogorsk, Chelyabinsk region, Russia $\left(52^{\circ} 42^{\prime} 00^{\prime \prime} \mathrm{N}, 60^{\circ} 26^{\prime} 27^{\prime \prime} \mathrm{E}\right)$

Elena V. Belogub*, Sergey N. Britvin, Vladimir V. Shilovskikh, Leonid A. Pautov, Vasiliy A. Kotlyarov and Elisaveta V. Zaykova ^E-mail: belogub_e@yahoo.com

The Se analogue of kingstonite

Monoclinic: $\mathrm{C} 2 / \mathrm{m}$; structure determined

$a=10.877(1), b=11.192(1), c=6.4796(6) \AA, \beta=108.887(2)^{\circ}$

5.43(37), 3.275(75), 3.199(100), 3.061(87), 2.568(62), 2.545(41), 3.413(34), 1.697(34)

Type material is deposited in the collections of the Fersman Mineralogical Museum, Russian Academy of Sciences, Leninskiy Prospekt 18-2, Moscow 119071, Russia, registration number 5395/1

How to cite: Belogub, E.V., Britvin, S.N., Shilovskikh, V.V., Pautov, L.A., Kotlyarov, V.A. and Zaykova, E.V. (2020) Zaykovite, IMA 2019-084. CNMNC Newsletter No. 54; Mineralogical Magazine, 84, https://doi.org/10.1180/mgm. 2020.21

\section{IMA No. 2019-110}

Oxycalciomicrolite

$\mathrm{Ca}_{2} \mathrm{Ta}_{2} \mathrm{O}_{7}$

Fumal pegmatite, Nazareno, Minas Gerais, Brazil $\left(21^{\circ}\right.$ $04^{\prime} 08.23^{\prime \prime} \mathrm{S}, 44^{\circ} 33^{\prime} 59.63^{\prime \prime} \mathrm{W}$ )

Victor Hugo Riboura Menezes da Silva*, Reiner Neumann, Ciro Alexandre Ávila, Fabiano Richard Leite Faulstich, Felipe Emerson André Alves and Filipe Barra de Almeida

*E-mail: vmenezes92@gmail.com

Pyrochlore supergroup

Cubic: $F d \overline{3} m$; structure determined

$a=10.3803(6) \AA$

6.023(89), 3.145(44), 3.011(100), 2.608(25), 2.008(14), 1.844 (48), 1.763(11), 1.573(33)

Type material is deposited in the mineralogical collections of the Museu Nacional, Universidade Federal do Rio de Janeiro, Quinta da Boa Vista s/n, 20940-040 Rio de Janeiro, Brazil, registration number $\mathrm{MN}-7601-\mathrm{M}$

How to cite: Menezes da Silva, V.H.R., Neumann, R., Ávila, C.A., Faulstich, F.R.L., Alves, F.E.A. and de Almeida, F.B. (2020) Oxycalciomicrolite, IMA 2019-110. CNMNC Newsletter No. 54; Mineralogical Magazine, 84, https://doi. org/10.1180/mgm.2020.21

\section{IMA No. 2019-113}

Uvite

$\mathrm{CaMg}_{3}\left(\mathrm{Al}_{5} \mathrm{Mg}\right)\left(\mathrm{Si}_{6} \mathrm{O}_{18}\right)\left(\mathrm{BO}_{3}\right)_{3}(\mathrm{OH})_{3}(\mathrm{OH})$

Facciatoia quarry, S. Piero in Campo (LI), Elba, Tuscany, Italy $\left(42^{\circ} 45^{\prime} 04.55^{\prime \prime} \mathrm{N}, 10^{\circ} 12^{\prime} 50.89^{\prime \prime} \mathrm{E}\right)$

Ferdinando Bosi*, Cristian Biagioni, Federico Pezzotta, Henrik Skobgy, Ulf Hålenius, Jan Cempírek, Frank C. Hawthorne, Aaron J. Lussier, Yassir A. Abdu, Maxwell C. Day, Mostafa Fayek, Christine M. Clark, Joel D. Grice and Darrell J. Henry
^E-mail: ferdinando.bosi@uniroma1.it

Tourmaline supergroup

Trigonal: $R 3 m$; structure determined $a=15.952(1), c=7.2222(5) \AA$

4.237(49), 3.994(51), 3.497(57), 2.973(88), 2.584(100), 2.047 (53), 1.925(37), 1.666(26)

Type material is deposited in the mineralogical collections of the Museo Civico di Storia Naturale, Corso Venezia 55, 20121 Milano, Italy, catalogue number M38848, and the Museo di Storia Naturale, Università di Pisa, Via Roma 79, 56011 Calci (PI), Italy, catalogue number 19911

How to cite: Bosi, F., Biagioni, C., Pezzotta, F., Skobgy, H., Hålenius, U., Cempírek, J., Hawthorne, F.C., Lussier, A.J., Abdu, Y.A., Day, M.C., Fayek, M., Clark, C.M., Grice, J.D. and Henry, D.J. (2020) Uvite, IMA 2019-113. CNMNC Newsletter No. 54; Mineralogical Magazine, 84, https://doi. org/10.1180/mgm.2020.21

\section{IMA No. 2019-114}

Rhabdoborite-(Mo)

$\mathrm{Mg}_{12} \mathrm{Mo}_{1.33}^{6+} \mathrm{O}_{6}\left(\mathrm{BO}_{3}\right)_{6} \mathrm{~F}_{2}$

Arsenatnaya fumarole, second scoria cone of the Northern Breakthrough of the Great Tolbachik fissure eruption, Tolbachik volcano, Kamchatka, Far-Eastern Region, Russia $\left(55^{\circ} 41^{\prime} \mathrm{N}, 160^{\circ} 14^{\prime} \mathrm{E}, 1200 \mathrm{~m}\right.$ asl $)$

Igor V. Pekov*, Natalia V. Zubkova, Natalia N. Koshlyakova, Dmitry I. Belakovskiy, Atali A. Agakhanov, Sergey N. Britvin, Evgeny G. Sidorov and Dmitry Y. Pushcharovsky

${ }^{\star}$ E-mail: igorpekov@mail.ru

Isostructural with rhabdoborite-(V) and rhabdoborite-(W)

Hexagonal: $P 6_{3}$; structure determined

$a=10.6304(3), c=4.5637(2) \AA$

9.20(100), 5.312(32), 3.483(73), 2.769(70), 2.549(40), 2.228(72), $1.702(61), 1.475(37)$

Type material is deposited in the collections of the Fersman Mineralogical Museum, Russian Academy of Sciences, Leninskiy Prospekt 18-2, Moscow 119071, Russia, registration number 5464/1

How to cite: Pekov, I.V., Zubkova, N.V., Koshlyakova, N.N., Belakovskiy, D.I., Agakhanov, A.A., Britvin, S.N., Sidorov, E.G. and Pushcharovsky, D.Y. (2020) Rhabdoborite-(Mo), IMA 2019-114. CNMNC Newsletter No. 54; Mineralogical Magazine, 84, https://doi.org/10.1180/mgm.2020.21

IMA No. 2019-115

Popugaevaite

$\mathrm{Ca}_{3}\left[\mathrm{~B}_{5} \mathrm{O}_{6}(\mathrm{OH})_{6}\right] \mathrm{FCl}_{2} \cdot 8 \mathrm{H}_{2} \mathrm{O}$

International diamond mine, Internatsional'naya kimberlite pipe, Irelyakh river basin, $10 \mathrm{~km} \mathrm{SW}$ of the city of Mirny, Sakha Republic, Siberia, Russia

Igor V. Pekov*, Natalia V. Zubkova, Ilya I. Chaikovskiy, Nikita V. Chukanov, Dmitry I. Belakovskiy, Vasiliy O. Yapaskurt, Yana V. Bychkova, Dmitry A. Ksenofontov, Elena P. Chirkova, Sergey N. Britvin and Dmitry Y. Pushcharovsky

${ }^{\star}$ E-mail: igorpekov@mail.ru

The $\mathrm{F}$ analogue of brianroulstonite

Monoclinic: $P n$; structure determined

$a=8.705(1), b=8.102(1), c=14.812(2) \AA, \beta=91.367(7)^{\circ}$

8.12(100), 7.13(12), 4.058(27), 3.577(15), 2.936(10), 2.834(16), 2.283(10), 2.104(9)

Type material is deposited in the collections of the Fersman Mineralogical Museum, Russian Academy of Sciences, 
Leninskiy Prospekt 18-2, Moscow 119071, Russia, registration number 5465/1

How to cite: Pekov, I.V., Zubkova, N.V., Chaikovskiy, I.I., Chukanov, N.V., Belakovskiy, D.I., Yapaskurt, V.O., Bychkova, Y.V., Ksenofontov, D.A., Chirkova, E.P., Britvin, S.N. and Pushcharovsky, D.Y. (2020) Popugaevaite, IMA 2019-115. CNMNC Newsletter No. 54; Mineralogical Magazine, 84, https://doi.org/10.1180/mgm.2020.21

\section{IMA No. 2019-116}

Freitalite

$\mathrm{C}_{14} \mathrm{H}_{10}$

Königin Carola shaft, Freital, near Dresden, Saxony, Germany $\left(51^{\circ} 00^{\prime} 10^{\prime \prime} \mathrm{N}, 13^{\circ} 38^{\prime} 18^{\prime \prime} \mathrm{E}\right)$

Thomas Witzke*, Martin Schreyer, René Csuk and Herbert Pöllmann

${ }^{\star}$ E-mail: thomas.witzke@panalytical.com

Known synthetic analogue (anthracene)

Monoclinic: $P 2_{1} / a$; structure determined

$a=8.5572(9), b=6.0220(5), c=11.173(1) \AA, \beta=124.174(1)^{\circ}$

9.252(98), 4.877(19), 4.628(10), 4.587(100), 4.152(7), 3.538(27),

$3.434(22), 3.050(20)$

Type material is deposited in the mineralogical collections of the Technische Universität Bergakademie Freiberg, Akademiestrass 6, 09599 Freiberg, Germany, specimens No. MiSa72396 and MiSa84590

How to cite: Witzke, T., Schreyer, M., Csuk, R. and Pöllmann, H. (2020) Freitalite, IMA 2019-116. CNMNC Newsletter No. 54; Mineralogical Magazine, 84, https://doi.org/10.1180/mgm. 2020.21

\section{IMA No. 2019-117}

Shuiskite- $(\mathrm{Cr})$

$\mathrm{Ca}_{2} \mathrm{CrCr}_{2}\left[\mathrm{SiO}_{4}\right]\left[\mathrm{Si}_{2} \mathrm{O}_{6}(\mathrm{OH})\right](\mathrm{OH})_{2} \mathrm{O}$

Rudnaya underground chromite mine (level $280 \mathrm{~m}$ ), Glavnoe Saranovskoe deposit, Saranovskaya deposits, Sarany, Middle Urals, Russia

Inna Lykova*, Dmitry A. Varlamov, Nikita V. Chukanov, Igor V. Pekov, Dmitry I. Belakovskiy, Oleg K. Ivanov, Natalia V. Zubkova and Sergey N. Britvin

*E-mail: ilykova@nature.ca

Pumpellyite group

Monoclinic: $\mathrm{C} 2 / \mathrm{m}$; structure determined

$a=19.2436(6), b=5.9999(2), c=8.8316(3) \AA, \beta=97.833(3)^{\circ}$

4.759(34), 4.707(36), 3.783(75), 2.913(100), 2.755(52), 2.539 (48), 2.470(39), 1.602(35)

Type material is deposited in the collections of the Fersman Mineralogical Museum, Russian Academy of Sciences, Leninskiy Prospekt 18-2, Moscow 119071, Russia, registration number 5481/1, and the Canadian Museum of Nature, 240 McLeod Street, Ottawa, ON K2P 2R1, Canada, catalogue number CMNMC 87302

How to cite: Lykova, I., Varlamov, D.A., Chukanov, N.V., Pekov, I.V., Belakovskiy, D.I., Ivanov, O.K., Zubkova, N.V. and Britvin, S.N. (2020) Shuiskite-(Cr), IMA 2019-117. CNMNC Newsletter No. 54; Mineralogical Magazine, 84, https://doi.org/10.1180/mgm.2020.21

\section{IMA No. 2019-118}

Alexkuznetsovite-(Ce)

$\mathrm{Ce}_{2} \mathrm{Mn}\left(\mathrm{CO}_{3}\right)\left(\mathrm{Si}_{2} \mathrm{O}_{7}\right)$
Mochalin Log REE deposit, $14 \mathrm{~km} \mathrm{~N}$ of the city of Kyshtym, Chelyabinsk Oblast', Southern Urals, Russia $\left(55^{\circ} 48^{\prime} 42^{\prime \prime} \mathrm{N}, 60^{\circ}\right.$ $33^{\prime} 46^{\prime \prime} \mathrm{E}$ )

Anatoly V. Kasatkin*, Natalia V. Zubkova, Igor V. Pekov, Nikita V. Chukanov, Radek Škoda, Atali A. Agakhanov and Dmitriy I. Belakovskiy

${ }^{\star}$ E-mail: anatoly.kasatkin@gmail.com

The Ce analogue of alexkuznetsovite-(La)

Monoclinic: $P 2_{1} / c$; structure determined

$a=6.5764(4), b=6.7685(4), c=18.749(1) \AA, \beta=108.672(8)^{\circ}$

$4.145(35), 3.177(26), 2.893(100), 2.797(36), 2.027(24), 1.833$

(26), 1.808(25), 1.686(20)

Type material is deposited in the collections of the Fersman Mineralogical Museum, Russian Academy of Sciences, Leninskiy Prospekt 18-2, Moscow 119071, Russia, registration number $5416 / 3$

How to cite: Kasatkin, A.V., Zubkova, N.V., Pekov, I.V., Chukanov, N.V., Škoda, R., Agakhanov, A.A. and Belakovskiy, D.I. (2020) Alexkuznetsovite-(Ce), IMA 2019-118. CNMNC Newsletter No. 54; Mineralogical Magazine, 84, https://doi.org/10.1180/mgm. 2020.21

\section{IMA No. 2019-120}

Biagioniite

$\mathrm{Tl}_{2} \mathrm{SbS}_{2}$

Museum sample from Hemlo gold deposit, Marathon, Ontario, Canada

Luca Bindi ${ }^{\star}$ and Yves Moëlo

^E-mail: luca.bindi@unifi.it

Isostructural with dervillite

Monoclinic: $P c$; structure determined

$a=11.0895(9), b=14.3124(11), c=7.9352(6) \AA, \beta=96.230(8)^{\circ}$

3.79(60), 3.68(40), 3.56(100), 3.48(40), 3.37(75), 3.16(49), 3.08 (40), 3.03(60)

Type material is deposited in the mineralogical collections of the Museo di Storia Naturale, Università di Firenze, Via La Pira 4, I-50121, Firenze, Italy, catalogue number 46582/G

How to cite: Bindi, L. and Moëlo, Y. (2020) Biagioniite, IMA 2019-120. CNMNC Newsletter No. 54; Mineralogical Magazine, 84, https://doi.org/10.1180/mgm.2020.21

IMA No. 2019-121

Jasonsmithite

$\mathrm{Mn}_{4}^{2+} \mathrm{ZnAl}\left(\mathrm{PO}_{4}\right)_{4}(\mathrm{OH})\left(\mathrm{H}_{2} \mathrm{O}\right)_{7} \cdot 3.5 \mathrm{H}_{2} \mathrm{O}$

East dump of the Foote Lithium Company mine, Kings Mountain district, Cleveland Co., North Carolina, USA $\left(35^{\circ}\right.$ $12^{\prime} 40^{\prime \prime} \mathrm{N}, 81^{\circ} 21^{\prime} 20^{\prime \prime} \mathrm{W}$ )

Anthony R. Kampf*, Aaron J. Celestian and Barbara P. Nash ^E-mail: akampf@nhm.org

New structure type

Monoclinic: $P 2_{1} / c$; structure determined

$a=8.5822(3), b=13.1770(6), c=20.304(1) \AA, \beta=98.485(7)^{\circ}$

10.98(43), 10.08(100), 7.95(18), 4.074(19), 3.029(30), 2.846(18), 2.605(29), 2.543(24)

Type material is deposited in the mineralogical collections of the Natural History Museum of Los Angeles County, 900 Exposition Boulevard, Los Angeles, CA 90007, USA, catalogue numbers 74374 (holotype), 74375 (cotype) and 74376 (cotype) How to cite: Kampf, A.R., Celestian, A.J. and Nash, B.P. (2020) Jasonsmithite, IMA 2019-121. CNMNC Newsletter No. 54; Mineralogical Magazine, 84, https://doi.org/10.1180/mgm. 2020.21 
IMA No. 2019-122

Johanngeorgenstadtite

$\mathrm{Ni}_{4.5}^{2+}\left(\mathrm{AsO}_{4}\right)_{3}$

Johanngeorgenstadt, Erzgebirgskreis, Saxony, Germany

Anthony R. Kampf*, Barbara P. Nash and Jakub Plášil

${ }^{\star}$ E-mail: akampf@nhm.org

Alluaudite supergroup

Monoclinic: $C 2 / c$; structure determined

$a=11.993(3), b=12.753(3), c=6.696(2) \AA, \beta=113.302(8)^{\circ}$

$3.514(16), 3.215(48), 3.076(18), 2.748(100), 2.623(20), 1.948$

(17), 1.661(28), 1.585(16)

Type material is deposited in the mineralogical collections of the American Museum of Natural History, Central Park West @ 79th Street, New York City, NY 10024, USA, catalogue number 17956

How to cite: Kampf, A.R., Nash, B.P. and Plášil, J. (2020) Johanngeorgenstadtite, IMA 2019-122. CNMNC Newsletter No. 54; Mineralogical Magazine, 84, https://doi.org/10.1180/ mgm.2020.21

\section{IMA No. 2019-123}

Grguricite

$\mathrm{CaCr}_{2}\left(\mathrm{CO}_{3}\right)_{2}(\mathrm{OH})_{4} \cdot 4 \mathrm{H}_{2} \mathrm{O}$

Adeghoual mine, northern outskirts of the town of Mibladen, Midelt region, Morocco $\left(32^{\circ} 46^{\prime} 00^{\prime \prime} \mathrm{N}, 4^{\circ} 37^{\prime} 59^{\prime \prime} \mathrm{W}\right)$

Mike S. Rumsey*, Mark D. Welch, John Spratt and Annette Kleppe

*E-mail: m.rumsey@nhm.ac.uk

The $\mathrm{Cr}$ analogue af alumohydrocalcite

Triclinic: $\mathrm{P} \overline{1}$

$a=5.724(2), \quad b=6.5304(9), \quad c=14.646(4) \AA, \quad \alpha=81.682(1)$,

$\beta=83.712(2), \gamma=86.365(2)^{\circ}$

7.208(45), 6.454(63), 6.222(100), 4.039(40), $3.227(87), 2.883$

(58), 2.648(47), 2.589(64)

Type material is deposited in the mineralogical collections of the Natural History Museum, Cromwell Road, London SW7 5BD, United Kingdom, specimen \# BM2019,5

How to cite: Rumsey, M.S., Welch, M.D., Spratt, J. and Kleppe, A. (2020) Grguricite, IMA 2019-123. CNMNC Newsletter No. 54; Mineralogical Magazine, 84, https://doi.org/10.1180/mgm. 2020.21

\section{IMA No. 2019-124}

Chukotkaite

$\mathrm{AgPb}_{7} \mathrm{Sb}_{5} \mathrm{~S}_{15}$

In the valley of Levyi Vulvyveem river, Amguema river basin, Iultin District, Chukotka Autonomous Okrug, Russia

Anatoly V. Kasatkin^, Jakub Plášil, Emil Makovicky, Radek Škoda, Atali A. Agakhanov, Ilya I. Chaikovskiy, Evgeny A. Vlasov and Igor V. Pekov

*E-mail: anatoly.kasatkin@gmail.com

New structure type

Monoclinic: $P 2{ }_{1} / c$; structure determined

$a=4.0575(3), b=35.950(1), c=19.221(2) \AA, \beta=90.525(8)^{\circ}$

3.52(100), 3.38(50), 3.13(50), 2.96(30), 2.82(25), 2.26(15), 2.23

(15), 1.91(50)

Type material is deposited in the collections of the Fersman Mineralogical Museum, Russian Academy of Sciences, Leninskiy Prospekt 18-2, Moscow 119071, Russia, registration number 5467/1

How to cite: Kasatkin, A.V., Plášil, J., Makovicky, E., Škoda, R., Agakhanov, A.A., Chaikovskiy, I.I., Vlasov, E.A. and Pekov, I.V.
(2020) Chukotkaite, IMA 2019-124. CNMNC Newsletter No. 54; Mineralogical Magazine, 84, https://doi.org/10.1180/mgm. 2020.21

IMA No. 2019-125

Luboržákite

$\mathrm{Mn}_{2} \mathrm{AsSbS}_{5}$

Vorontsovskoe gold deposit, ca. $13 \mathrm{~km} \mathrm{~S}$ of the city of Krasnotur'insk, Sverdlovskaya Oblast', Northern Urals, Russia $\left(59^{\circ} 66^{\prime} 29^{\prime \prime} \mathrm{N}, 60^{\circ} 19^{\prime} 61^{\prime \prime} \mathrm{E}\right)$

Anatoly V. Kasatkin*, Jakub Plášil, Emil Makovicky, Radek Škoda, Atali A. Agakhanov and Sergey Y. Stepanov

*E-mail: anatoly.kasatkin@gmail.com

Pavonite homologous series

Monoclinic: $\mathrm{C} 2 / \mathrm{m}$; structure determined

$a=12.5077(6), b=3.8034(2), c=16.0517(8) \AA, \beta=94.190(4)^{\circ}$

$3.486(50), 3.286(50), 3.262(36), 2.806(39), 2.788(57), 2.690$

(100), 1.904(37), 1.902(36)

Type material is deposited in the collections of the Fersman Mineralogical Museum, Russian Academy of Sciences, Leninskiy Prospekt 18-2, Moscow 119071, Russia, registration number 5498/1

How to cite: Kasatkin, A.V., Plášil, J., Makovicky, E., Škoda, R., Agakhanov, A.A. and Stepanov, S.Y. (2020) Luboržákite, IMA 2019-125. CNMNC Newsletter No. 54; Mineralogical Magazine, 84, https://doi.org/10.1180/mgm.2020.21

IMA No. 2019-126

Cuyaite

$\mathrm{Ca}_{2} \mathrm{Mn}^{3+} \mathrm{As}_{14}^{3+} \mathrm{O}_{24} \mathrm{Cl}$

NW side of the steep Camarones Valley, ca. $9 \mathrm{~km}$ NE of the village of Cuya, Arica Province, Chile

Anthony R. Kampf*, Barbara Nash, Maurizio Dini and Arturo A. Molina Donoso

*E-mail: akampf@nhm.org

New structure type

Monoclinic: $P n$; structure determined

$a=14.7231(6), b=5.5871(2), c=17.418(1) \AA, \beta=112.451(8)^{\circ}$

4.73(45), 3.162(100), 3.035(28), 3.004(37), 2.931(90), 2.799(28),

1.854(23), $1.832(26)$

Type material is deposited in the mineralogical collections of the Natural History Museum of Los Angeles County, 900 Exposition Boulevard, Los Angeles, CA 90007, USA; catalogue number 74462

How to cite: Kampf, A.R., Nash, B., Dini, M. and Molina Donoso, A.A. (2020) Cuyaite, IMA 2019-126. CNMNC Newsletter No. 54; Mineralogical Magazine, 84, https://doi. org/10.1180/mgm.2020.21

\section{IMA No. 2019-127}

Brattforsite

$\mathrm{Mn}_{19} \mathrm{As}_{12} \mathrm{O}_{36} \mathrm{Cl}_{2}$

Brattforsgruvan mine, Filipstad municipality, Värmland, Sweden $\left(59^{\circ} 49^{\prime} 59^{\prime \prime} \mathrm{N}, 14^{\circ} 07^{\prime} 25^{\prime \prime} \mathrm{E}, 230 \mathrm{~m}\right.$ asl)

Dan Holtstam*, Cristian Biagioni and Ulf Hålenius

${ }^{*}$ E-mail: dan.holtstam@nrm.se

The $\mathrm{Cl}$ analogue of magnussonite, with lower symmetry Monoclinic: $C 2 / c$; structure determined

$a=27.7223(9), b=19.5763(7), c=19.5806(7) \AA, \beta=134.541(1)^{\circ}$

2.843(100), 2.828(99), 2.471(16), 2.448(28), 1.746(16), 1.739

(25), 1.731(32), 1.475(16) 
Type material is deposited in the mineralogical collections of the Department of Geosciences, Swedish Museum of Natural History, Box 50007, SE-10405 Stockholm, Sweden, collection number GEO-NRM \#19100303, and the Natural History Museum, University of Pisa, Via Roma 79, Calci (PI), Italy, catalogue number 19912

How to cite: Holtstam, D., Biagioni, C. and Hålenius, U. (2020) Brattforsite, IMA 2019-127. CNMNC Newsletter No. 54; Mineralogical Magazine, 84, https://doi.org/10.1180/mgm. 2020.21

\section{NOMENCLATURE/CLASSIFICATION PROPOSALS APPROVED IN FEBRUARY 2020}

\section{Arctite supergroup}

The arctite supergroup has been established; it currently includes nine mineral species. The supergroup is divided into the zadovite group (alternation of tetrahedral layers and antiperovskite layers TA) and the arctite group (alternation of tetrahedral layers and triple antiperovskite layers $-T A^{3}$ ).

\section{Fillowite group}

The fillowite group has been established; it currently includes four mineral species. The chemical formulae of the minerals of the fillowite group have been uniformed, based on $9\left(\mathrm{PO}_{4}\right)$ groups per formula unit, as follows: fillowite, $\mathrm{Na}_{3} \mathrm{CaMn}_{11}^{2+}\left(\mathrm{PO}_{4}\right)_{9}$; johnsomervilleite, $\mathrm{Na}_{3} \mathrm{CaFe}_{11}^{2+}\left(\mathrm{PO}_{4}\right)_{9}$; chladniite, $\mathrm{Na}_{3} \mathrm{CaMg}_{11}\left(\mathrm{PO}_{4}\right)_{9}$; galileiite, $\mathrm{Na}_{3} \mathrm{Fe}^{2+} \mathrm{Fe}_{11}^{2+}\left(\mathrm{PO}_{4}\right)_{9}$. Stornesite- $(\mathrm{Y})$ has been discredited as it is equivalent to $\mathrm{Y}$-bearing chladniite.

\section{Magnetoplumbite group}

The magnetoplumbite group has been established; it currently includes eleven mineral species. Minerals of the magnetoplumbite group have the general formula $A B_{12} \mathrm{O}_{19}$. The group is divided into subgroups, based on the dominant $A$ cation: magnetoplumbite subgroup $(A=\mathrm{Pb})$, hawthorneite subgroup $(A=\mathrm{Ba})$, and hibonite subgroup $(A=\mathrm{Ca})$. Hibonite- $(\mathrm{Fe})$ has been renamed to chihuahuaite.

\section{Walentaite group}

The walentaite group has been established; it currently includes four mineral species. Minerals of the walentaite group have the general formula $\left[\left(A 1, A 1^{\prime}, A 2, \square\right)\left(\mathrm{H}_{2} \mathrm{O}\right) n\right]\left[\mathrm{BxAs}_{3-x}^{3+} \mathrm{M} 1(\mathrm{M} 2)_{2}\left(\mathrm{TO}_{4}\right)_{2}\right.$ $\left.(\mathrm{O}, \mathrm{OH})_{7}\right]$. The group is divided into subgroups based on the dominant $T$ cation: walentaite subgroup $(T=\mathrm{P})$, and halilsarpite subgroup $(T=\mathrm{As})$.

\section{NOMENCLATURE/CLASSIFICATION PROPOSALS APPROVED IN MARCH 2020}

\section{IMA 19-I: Redefinition of fairbankite}

Proposal 19-I is accepted, and fairbankite is redefined. The previously accepted formula was $\mathrm{Pb}^{2+} \mathrm{Te}^{4+} \mathrm{O}_{3}$. Now it has been proven that the sulfate group is an essential constituent and the formula has been changed to $\mathrm{Pb}_{12}^{2+}\left(\mathrm{Te}^{4+} \mathrm{O}_{3}\right)_{11}\left(\mathrm{SO}_{4}\right)$.

\section{Renaming of shuiskite}

After the approval of shuiskite-(Cr) (IMA No. 2019-117; see above), and considering that the nomenclature of the pumpellyite group is based on suffixes, it was decided to change the name of the mineral shuiskite to shuiskite-(Mg). The rationale in the nomenclature of the pumpellyite group is that the root-name depends on the dominant - always trivalent - cation at $M 2$, whereas the suffix depends on the dominant cation at $M 1$. Currently the root-names are as follows: pumpellyite $(M 2=\mathrm{Al})$, julgoldite $\left(M 2=\mathrm{Fe}^{3+}\right)$, shuiskite $(M 2=\mathrm{Cr})$; okhotskite $\left(M 2=\mathrm{Mn}^{3+}\right)$, poppiite $\left(M 2=\mathrm{V}^{3+}\right)$. The valid species are as follows: pumpellyite-( $\mathrm{Mg})$, pumpellyite- $\left(\mathrm{Fe}^{2+}\right)$, pumpellyite- $\left(\mathrm{Fe}^{3+}\right)$, pumpellyite- $\left(\mathrm{Mn}^{2+}\right)$, pumpellyite-( $\left.\mathrm{Al}\right)$, julgoldite- $\left(\mathrm{Fe}^{2+}\right)$, julgoldite$\left(\mathrm{Fe}^{3+}\right)$, julgoldite- $(\mathrm{Mg})$, shuiskite- $(\mathrm{Mg})$, shuiskite- $(\mathrm{Cr})$, okhotskite and poppiite. The latter two minerals are (still) without suffix as they stand alone with their root-name. 